\title{
KDamping: A stiffness based vibration absorption concept
}

\author{
loannis A Antoniadis, Professor, antogian@central.ntua.gr \\ Mechanical Design and Control Systems Section, \\ Mechanical Engineering Department \\ National Technical University of Athens, \\ Heroon Polytechniou 9, 15780 Zografou, Greece
}

\begin{abstract}
The KDamper is a novel passive vibration isolation and damping concept, based essentially on the optimal combination of appropriate stiffness elements, which include a negative stiffness element. The KDamper concept does not require any reduction in the overall structural stiffness, thus overcoming the corresponding inherent disadvantage of the "Quazi Zero Stiffness” (QZS) isolators, which require a drastic reduction of the structure load bearing capacity. Compared to the traditional Tuned Mass damper (TMD), the KDamper can achieve better isolation characteristics, without the need of additional heavy masses, as in the case of TMD. Contrary to the TMD and the inerter, the KDamper substitutes the necessary high inertial forces of the added mass by the stiffness force of the negative stiffness element. Among others, this can provide comparative advantages in the very low frequency range.

The paper proceeds to a systematic analytical approach for the optimal design and selection of the parameters of the KDamper, following exactly the classical approach used for the design of the TMD. It is thus theoretically proved, that the KDamper can inherently offer far better isolation and damping properties than the TMD. Moreover, since the isolation and damping properties of the KD essentially result from the stiffness elements of the system, further technological advantages can emerge, in terms of weight, complexity and reliability.

A simple vertical vibration isolation example is provided, implemented by a set of optimally combined conventional linear springs. The system is designed so that the system presents an adequate static load bearing capacity, while the transfer function of the system is below unity in the entire frequency range. Further insight is provided to the physical behaviour of the system, indicating a proper phase difference between the positive and the negative stiffness elastic forces. This fact ensures that an adequate level of elastic forces exists throughout the entire frequency range, able to counteract the inertial and the external excitation forces, while the damping forces and the inertia forces of the additional mass remain minimal in the entire frequency range, including the natural frequencies.
\end{abstract}

\section{Introduction}

Among the established vibration absorption concepts, the Tuned Mass Damper has the longest history. A tuned mass damper (TMD) is a device consisting of a mass, a spring, and a damper that is attached to a structure in order to reduce the dynamic response of the structure, by modifying its transfer function close to the original structure natural frequency(ies). The TMD concept was first 
applied by Frahm in 1909 [1]. A theory for the TMD was presented later in the paper by Ormondroyd and Den Hartog [2] . A detailed discussion of optimal tuning and damping parameters appears in Den Hartog's book on mechanical vibrations [3]. Since then, numerous applications of various forms of TMDs have been reported. Some recent examples include vibration absorption in seismic or other forms of excitation of structures [4], wind and wave excitation in wind turbines [5] and tortional vibrations in rotating and reciprocating machines [6]. TMDs are available in various physical forms, including solids, liquids [5], or even active implementations [7]. The essential limitation of the TMD is that a large oscillating mass is required in order to achieve significant vibration reduction. Among others, this has prohibited the usage of TMDs in the automotive or aerospace sector.

In an attempt to reduce the requirements for heavy oscillating masses, the inerter concept has been introduced in early 2000s by Smith [8]. The inerter is a two terminal element which has the property that the force generated at its ends is proportional to the relative acceleration of its terminals. Its constant of proportionality is called inertance and is measured in kilograms. The main advantage of the inerter is that the inerter need not have large mass in order to achieve the same inertia effect as the additional mass of the TMD. However, since inerters, dampers and springs can be connected in multiple configurations [9], the comparison of the structure of the Frequency Response functions of the inerter and of the TMD becomes very complicated. In 2005 the inerter was profitably used as a part of suspension in Formula 1 racing car under the name of J-damper [10]. Since then, other applications emerged, such as in suspensions of railway vehicles [11] or in seismic protection of structures [12]. Although the initial inerter configuration is for linear accelerations, rotary versions [13], or even active configurations [14] have been proposed. Still, to work efficiently, all considered devices have to be precisely tuned which can be hard to achieve or even impossible in some cases. Moreover proposed TMDs with inerters suffer from susceptibility to detuning. Although variable inertance mechanical configurations have been proposed for this purpose [15], the essential limitation of the inerter is the complex and elaborate mechanical design configurations needed for its implementation.

A parallel direction to the above is the concept of introducing negative stiffness elements (or 'anti-springs') for vibration isolation. This concept has also a long history, being first introduced in the pioneering publication of Molyneaux [16], as well as in the milestone developments of Platus [17]. The central concept of these approaches is to significantly reduce the stiffness of the isolator and consequently to reduce the natural frequency of the system even at almost zero levels [18], being thus called "Quazi Zero Stiffness" (QZS) oscillators. In this way, the transmissibility of the system for all operating frequencies above the natural 
frequency is reduced, resulting to enhanced vibration isolation. An initial comprehensive review of such designs can be found in [19].

The negative stiffness behavior is primarily achieved by special mechanical designs involving conventional positive stiffness pre-stressed elastic mechanical elements, such as post-buckled beams, plates, shells and pre-compressed springs, arranged in appropriate geometrical configurations. Some interesting designs are described in [20],[21]. However, alternatively to elastic forces, other forms of physical forces can be used to produce an equivalent negative stiffness effect, such as gravitational [22], magnetic [23] or electromagnetic [24]. Quazi Zero Stiffness (QZS) oscillators are finding numerous applications in seismic isolation $[25,26,27,28]$, all types of automotive suspensions [29-31] or tortional vibrations [32].

Quite recently, periodic cellular structures with advanced dynamic behavior have been also proposed [33,34,35,36], combining high positive and negative stiffness. Although the physical mechanisms that generate increased damping in cellular structures are not well understood, microbuckling or slip- stick phenomena $[37,38,39]$ could be among the possible explanations.

Parallel, quite interesting possibilities towards achieving significant damping have been demonstrated to exist also in materials comprising a negative stiffness phase [40], not only at a material level [41], but also at macroscopic devices [42]. Quite interestingly, such a behaviour is combined with high stiffness properties. A theoretical approach has been performed for the analysis of the static and dynamic stability of composite materials, incorporating negative stiffness elements [43].

However, Quazi Zero Stiffness (QZS) oscillators suffer from their fundamental requirement for a drastic reduction of the stiffness of the structure almost to negligible levels, which limits the static load capacity of such structures.

It should be noted that a rich variety of nonlinear dynamic phenomena $[44,45,46,47,48,49]$, either inherently present, or designed to be present in all types of the above vibration absorbers, greatly contribute to the complexity of their dynamic behavior, as well as to the increase of their dynamic performance.

Quite recently, a novel type of oscillator has been proposed [50], incorporating a negative stiffness element, which can exhibit extraordinary damping properties, without presenting the drawbacks of the traditional linear oscillator, or of the 'zero-stiffness' designs. This oscillator is designed to present the same overall (static) stiffness as a traditional reference original oscillator. However, it differs both from the original SDoF oscillator, as well as from the known negative stiffness oscillators, by appropriately redistributing the individual stiffness elements and by reallocating the damping. Although the proposed oscillator incorporates a negative stiffness element, it is designed to be both statically and dynamically stable. Once such a system is 
designed according to the approach proposed in [50], it is shown to exhibit an extraordinary damping behaviour. Moreover, a drastic increase of several orders of magnitude has been observed for the damping ratio of the flexural waves propagating within layered periodic structures incorporating such negative stiffness oscillators [51].

In this paper, the concept of [50] is treated in a systematic way, within the general context of the design of the general class of tuned mass dampers. Section 2 of the paper performs an overview of the dynamic structure of the main conventional vibration absorbers/isolators -the Quazi Zero Stiffness Oscillator, the Tuned Mass damper and the Inerter- together with a concise presentation of their disadvantages. Next the KDamper is introduced, together with a preliminary conceptual presentation on its fundamental concept and on why this concept can overcome the disadvantages of the traditional vibration absorbers. The optimal selection approach of the KDamper parameters is introduced in Section 3, which follows exactly the same steps of [3]. In this way, a direct comparison of the KDamper with the TMD damper is performed in section 4, which reveals its basic properties. The KDamper always indicates a better isolation properties than a TMD damper with the same additional mass. Instead of increasing the additional mass, the vibration isolation capability of the KDamper can be increased by increasing the value of the negative stiffness element. Consequently, significant vibration isolation properties can be achieved, even for very low values (practically insignificant) of the additional mass. However, the increase of the negative stiffness element is upper bounded by the static stability limit of the structure.

Section 5 presents an application of the KDamper concept. The linear negative stiffness element is realized by a non-linear bistable element, which operates around an unstable equilibrium point. This bistable element takes the form of two horizontal springs, which transfer a negative force to a vertical spring through an appropriate mechanism. The system is designed so that it presents an adequate static load bearing capacity, while the transfer function of the system is below unity in the entire frequency range.The stiffness elements of this oscillator are selected according to the design procedure analysed in section 3 .

Further insight to the physical behavior of the system is provided, by analyzing the balance of forces in the entire frequency range. It is observed that the magnitude of the damping force and of the inertia force of the additional mass is minimal throughout the entire frequency range, including the natural frequencies of the system. Thus, the external force is almost entirely balanced by the positive and negative stiffness forces, as well as by the inertia of the main mass. This fact ensures that an adequate level of elastic forces exists throughout the entire frequency range, able to counteract the inertial and the external excitation forces and justifies the concept of stiffness based vibration isolation. 


\section{Overview of conventional vibration absorbers and the KDamper concept}

Figure 1 presents the basic layout of the fundamental vibration isolation and damping concepts to be considered. They are all designed to minimize the response $x(t)$ of an undamped SDoF system of mass $m$ and total static stiffness $k$ of to an external excitation force $f(t)$.

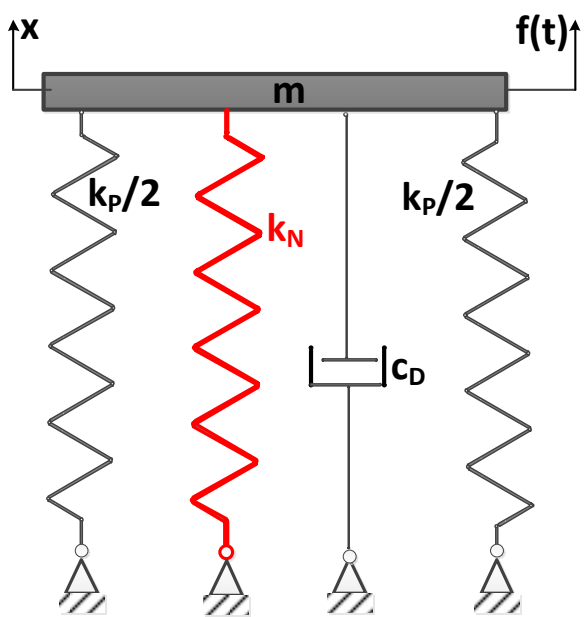

(a)

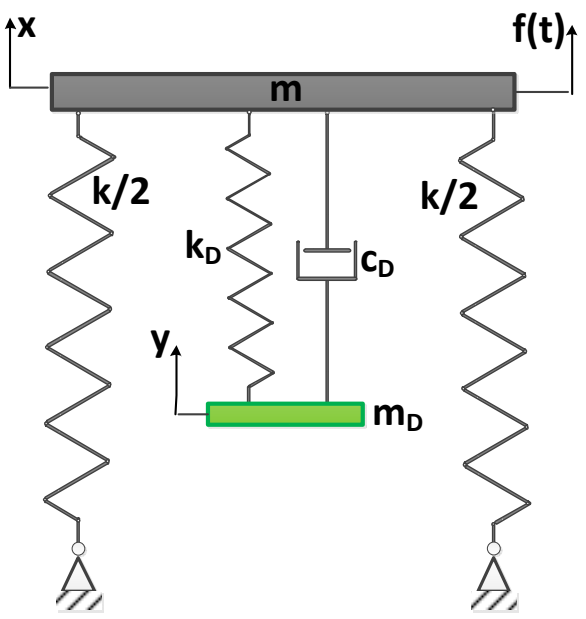

(b)

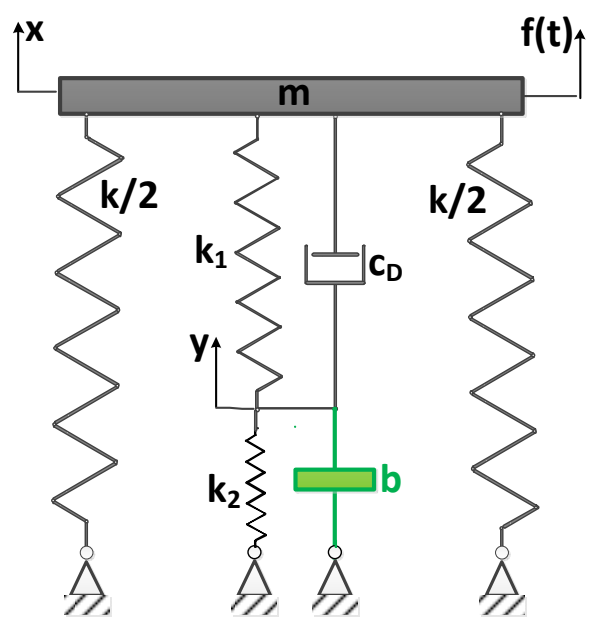

(c)

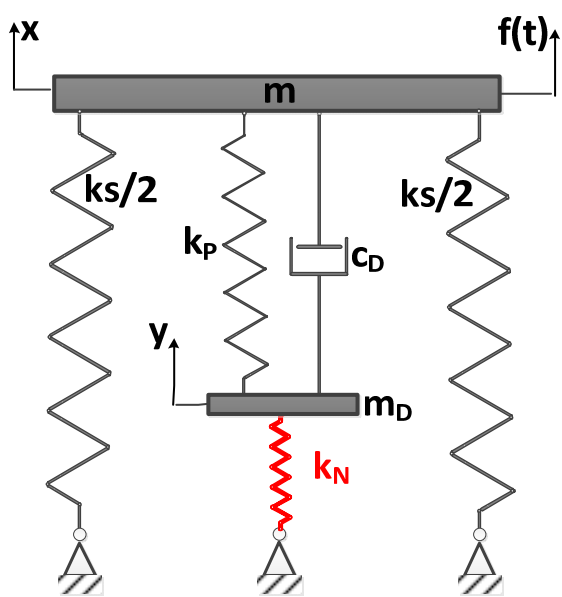

(d)

Figure 1. Schematic presentation of the main vibration isolation and damping concepts (a) Quasi-Zero Stiffness (QZS) oscillator, (b) Tunned Mass Damper(TMD), (c) Inerter (JDamper), (d) KDamper. 
The concept of the QZS oscillator is to add a negative stiffness element $k_{N}$ in parallel to the conventional positive stiffness element $k_{P}$. The equation of motion of the Quazi Zero Stiffness (QZS) oscillator is :

$$
m \ddot{x}+c_{D} \dot{x}+\left(k_{P}+k_{N}\right) x=m \ddot{x}+c_{D} \dot{x}+k x=f
$$

Since $k_{N}$ is negative, the overall static stiffness $k=k_{N}+k_{P}$ of the system is reduced. This correspondingly reduces the natural frequency $f_{0}$ of the system.

$$
f_{0}=\frac{1}{2 \pi} \sqrt{\frac{k}{m}}
$$

However, this limits the static loading capacity of the structure, which may result to unsolvable problems, especially in vertical vibration isolation. For example, if $X_{V S D}$ denotes the static deflection of such an isolation system under its own weight in the vertical direction:

$$
X_{V S D}=\frac{m g}{k}
$$

the combination of equations (2) and (3) leads to:

$$
X_{V S D}=\frac{g}{\left(2 \pi f_{0}\right)^{2}}
$$

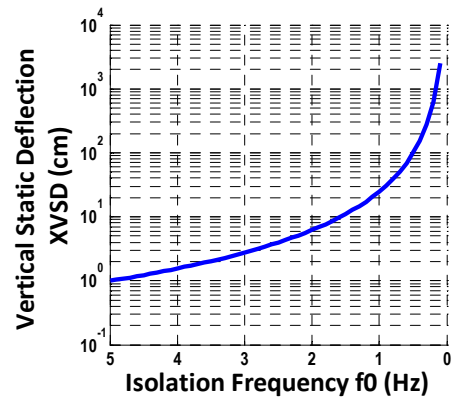

Figure 2: Dependence of static deflection on the isolation frequency for vertical vibration isolation

Fig. 2 presents graphically eq (4) and it clearly reflects the implicit constraints for low frequency vibration isolation, especially in the vertical direction. It should be noted that according to Eq. (4), $\mathrm{X}_{S V D}$ depends only on the gravity and the natural frequency $f_{0}$.

The equation of motion of the Tuned Mass Damper (TMD) is:

$m \ddot{x}+c_{D}(\dot{x}-\dot{y})+k x+k_{D}(x-y)=f$

$m_{D} \ddot{y}-c_{D}(\dot{x}-\dot{y})-k_{D}(x-y)=0$

or equivalently: 
$m \ddot{x}+k x+m_{D} \ddot{y}=f$

$m_{D} \ddot{y}-c_{D}(\dot{x}-\dot{y})-k_{D}(x-y)=0$

Assuming a harmonic excitation in the form of:

$f(t)=k X_{S T} \exp (j \omega t)$

and a steady state response of:

$x(t)=\tilde{X} \exp (j \omega t)$

$y(t)=\tilde{Y} \exp (j \omega t)$

where $\tilde{X}, \tilde{Y}$ denote the complex response amplitudes, the equations of motion (5) of the TMD become:

$-\omega^{2} m \tilde{X}+j \omega c_{D}(\tilde{X}-\tilde{Y})+k \tilde{X}+k_{D}(\tilde{X}-\tilde{Y})=k X_{S T}$

$-\omega^{2} m_{D} \tilde{Y}-j \omega c_{D}(\tilde{X}-\tilde{Y})-k_{D}(\tilde{X}-\tilde{Y})=0$

or equivalently:

$-\omega^{2} m \tilde{X}+k \tilde{X}-\omega^{2} m_{D} \tilde{Y}=k X_{S T}$

$-\omega^{2} m_{D} \tilde{Y}-j \omega c_{D}(\tilde{X}-\tilde{Y})-k_{D}(\tilde{X}-\tilde{Y})=0$

The resulting transfer function for the response amplitude $\tilde{X}$ is:

$\widetilde{T}_{X M}=\frac{\tilde{X}}{X_{S T}}=\frac{\left(-\omega^{2} m_{D}+j \omega c_{D}+k_{D}\right) k}{\left(-\omega^{2} m+j \omega c_{D}+k+k_{D}\right)\left(-\omega^{2} m_{D}+j \omega c_{D}+k_{D}\right)-\left(j \omega c_{D}+k_{D}\right)^{2}}$

or

$\tilde{T}_{X M}=\frac{\tilde{X}}{X_{S T}}=\frac{\left(\rho^{2}-q^{2}\right)+j 2 \zeta_{D} \rho q}{\left[q^{4}-q^{2}\left(1+\rho^{2}+\mu \rho^{2}\right)+\rho^{2}\right]+j 2 \zeta_{D} \rho q\left[1-q^{2}(1+\mu)\right]}$

where:

$q=\omega / \omega_{0}$

$\rho=\omega_{D} / \omega_{0}$

$\omega_{0}=\sqrt{k / m}$

$\omega_{D}=\sqrt{k_{D} / m_{D}}$

$\zeta_{D}=c_{D} / 2 \sqrt{k_{D} m_{D}}$

$\mu=m_{D} / m$

An approach for the optimal selection of the TMD parameters $\rho$ and $\zeta_{D}$ (optimal "TMD tuning") can be found among others in [3] and it leads to the following results: 


$$
\begin{aligned}
& \rho_{O P T}=1 /(1+\mu) \\
& \zeta_{D_{O P T}}=\sqrt{3 \mu / 8(1+\mu)^{3}}
\end{aligned}
$$

The maximum value of the amplitude of the transfer function becomes:

$$
T_{X M \max }=\frac{X_{M A X}}{X_{S T}}=\frac{|\tilde{X}|_{M A X}}{X_{S T}}=\sqrt{\frac{2+\mu}{\mu}}
$$

Figure 3 presents the effect of the mass ratio $\mu$ on the Transfer function of the TMD. A $\varsigma$ it can be noticed, a high value of $\mu$ is required for efficient vibration isolation, a fact which consists the major disadvantage of the TMD.

An indicative form of the implementation of the inerter is presented in Fig 1.c. It corresponds to a simplified version of the configuration A of [9].

The equation of motion of this specific configuration of the Inerter is:

$$
\begin{aligned}
& m \ddot{x}+c_{D}(\dot{x}-\dot{y})+k x+k_{1}(x-y)=f \\
& b \ddot{y}-c_{D}(\dot{x}-\dot{y})-k_{1}(x-y)+k_{2} y=0
\end{aligned}
$$

From a dynamics point of view, the transfer function of the system of equations (13) for the inerter is exactly the same as the transfer function of the TMD in Eq (8), when the value of $k_{2}$ is equal to zero and for $b=m_{D}, k_{1}=K_{D}$. Moreover, as it will be further proven in chapter 4, a positive value for $k_{2}$ has an adverse effect to the transfer function of the system. Therefore, the main advantage of the inerter over the TMD is considered to be technological: due to the technological design of the inerter, it can achieve the same inertia effect with a TMD, while it requires significantly less added mass than the TMD.

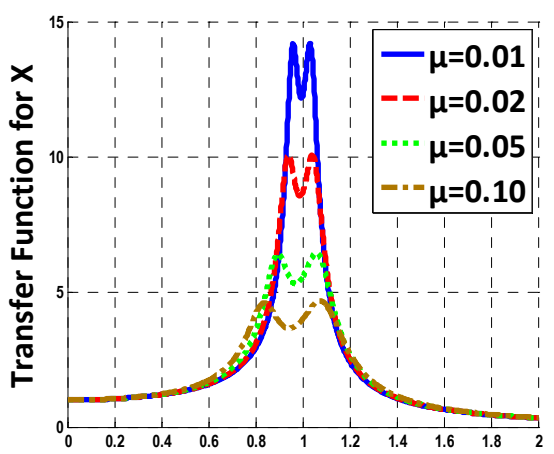

Relative frequency q

(a)

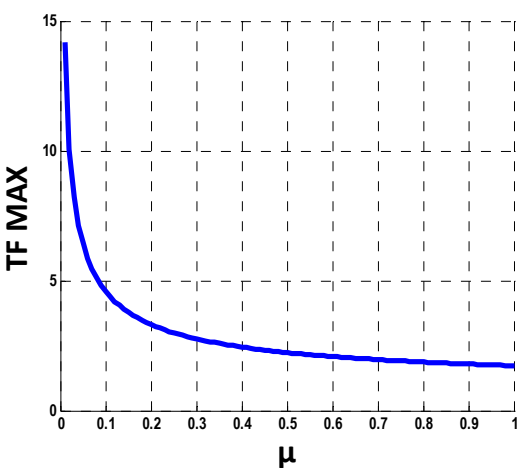

(b)

Figure 3: Effect of the mass ratio $\mu$ on the Transfer function of the TMD (a)Values for four different values of $\mu$. (b) variation of the maximum value of the transfer function over $\mu$. 
Figure 1.d presents the fundamental concept of the KDamper. Similarly to the QZS oscillator, it uses a negative stiffness element $k_{N}$. However, contrary to the QZS oscillator, the first basic requirement of the KDamper is that the overall static stiffness of the system is maintained:

$k_{S}+\frac{k_{P} k_{N}}{k_{P}+k_{N}}=k$

In this way, the KDamper can overcome the fundamental disadvantage of the QZS oscillator. Compared to the TMD damper, and similarly to the inerter, the KDamper also uses a dual port element connects the additional mass also to the base. However, instead of an inertial element (i.e. the inerter), the KDamper uses a negative stiffness element $k_{N}$. Thus, the equation of motion of the KDamper becomes:

$$
\begin{aligned}
& m \ddot{x}+c_{D}(\dot{x}-\dot{y})+k_{S} x+k_{P}(x-y)=f \\
& m_{D} \ddot{y}-c_{D}(\dot{x}-\dot{y})-k_{P}(x-y)+k_{N} y=0
\end{aligned}
$$

or equivalently:

$$
\begin{aligned}
& m \ddot{x}+k_{S} x+m_{D} \ddot{y}+k_{N} y=f \\
& m_{D} \ddot{y}-c_{D}(\dot{x}-\dot{y})-k_{P}(x-y)+k_{N} y=0
\end{aligned}
$$

Assuming a harmonic excitation in the form of eq (6) and corresponding harmonic responses in the form of eq (7), the equations of motion (15) of the TMD become:

$$
\begin{aligned}
& -\omega^{2} m \tilde{X}+j \omega c_{D}(\tilde{X}-\tilde{Y})+k_{S} \tilde{X}+k_{P}(\tilde{X}-\tilde{Y})=k X_{S T} \\
& -\omega^{2} m_{D} \tilde{Y}-j \omega c_{D}(\tilde{X}-\tilde{Y})-k_{P}(\tilde{X}-\tilde{Y})+k_{N} \tilde{Y}=0
\end{aligned}
$$

or equivalently:

$$
\begin{aligned}
& -\omega^{2} m \tilde{X}+k_{S} \tilde{X}-\omega^{2} m_{D} \tilde{Y}+k_{N} \tilde{Y}=k X_{S T} \\
& -\omega^{2} m_{D} \tilde{Y}-j \omega c_{D}(\tilde{X}-\tilde{Y})-k_{P}(\tilde{X}-\tilde{Y})+k_{N} \tilde{Y}=0
\end{aligned}
$$

A careful examination of eqs (16.c) reveals that the amplitude $F_{M D}$ of the inertia force of the additional mass and the amplitude $F_{N}$ of the negative stiffness force:

$$
\begin{aligned}
& F_{M D}=-\omega^{2} m_{D} Y \\
& F_{N}=k_{N} Y
\end{aligned}
$$

are exactly in phase, due to the negative value of $k_{N}$. Thus, similarly to the inerter, the KDamper essentially consists an indirect approach to increase the inertia effect of the additional mass $m_{D}$. without however increasing the mass $m_{D}$ itself. This is more obvious by a further comparison of eqs (8.c) and (16.c). Moreover, it should be noticed that the value of $F_{M D}$ depends on the frequency, while the value of $F_{N}$ is constant in the entire frequency range, a fact which is of importance for low frequency vibration isolation. 


\section{Optimal design approach for the KDamper}

The optimal design approach for the parameters of the $\mathrm{KD}$ damper follows exactly the corresponding steps as in [3]. First, the transfer function of the KD damper results from eqs (16).

$$
\begin{aligned}
& \widetilde{T}_{X K}=\frac{\tilde{X}}{X_{S T}}=\frac{\left(-\omega^{2} m_{D}+j \omega c_{D}+k_{D}\right) k}{\left(-\omega^{2} m+j \omega c_{D}+k_{S}+k_{P}\right)\left(-\omega^{2} m_{D}+j \omega c_{D}+k_{D}\right)-\left(j \omega c_{D}+k_{P}\right)^{2}} \\
& \widetilde{T}_{Y K}=\frac{\tilde{Y}}{\widetilde{X}} \widetilde{T}_{X K}=\frac{\left(j \omega c_{D}+k_{P}\right) k}{\left(-\omega^{2} m_{D}+j \omega c_{D}+k_{D}\right)} \widetilde{T}_{X K}
\end{aligned}
$$

where:

$k_{D}=k_{P}+k_{N}$

and $k$ is defined in (14).

In view of the fact that $k_{N}$ is negative and $k_{D}$ is positive, the parameter $\kappa$ is defined as:

$\kappa=-k_{N} / k_{D}=-k_{N} /\left(k_{P}+k_{N}\right)$

Eq (18) becomes:

$\tilde{T}_{X K}=\frac{\tilde{X}}{X_{S T}}=\frac{A+\left(j 2 \zeta_{D}\right) B}{C+\left(j 2 \zeta_{D}\right) D}$

$\widetilde{T}_{Y X K}=\frac{\tilde{Y}}{\widetilde{X}}=\frac{E+\left(j 2 \zeta_{D}\right) B}{A+\left(j 2 \zeta_{D}\right) B}$

and consequently:

$T_{X K}=\frac{|\tilde{X}|}{X_{S T}}=\frac{X}{X_{S T}}=\sqrt{\frac{A^{2}+\left(2 \zeta_{D}\right)^{2} B^{2}}{C^{2}+\left(2 \zeta_{D}\right)^{2} D^{2}}}$

$T_{Y X K}=\frac{Y}{X}=\sqrt{\frac{E^{2}+\left(2 \zeta_{D}\right)^{2} B^{2}}{A^{2}+\left(2 \zeta_{D}\right)^{2} B^{2}}}$

$T_{Y K}=\frac{Y}{X_{S t}}=\frac{Y}{X} \frac{X}{X_{S T}}=\sqrt{\frac{E^{2}+\left(2 \zeta_{D}\right)^{2} B^{2}}{C^{2}+\left(2 \zeta_{D}\right)^{2} D^{2}}}$

where:

$A=\rho^{2}-q^{2}$

$B=\rho q$

$C=q^{4}-q^{2}\left[1+\rho^{2}+(1+\kappa)^{2} \mu \rho^{2}\right]+\rho^{2}$

$D=\rho q\left[\left(1+\kappa^{2} \mu \rho^{2}\right)-q^{2}(1+\mu)\right]$ 


$$
E=(1+\kappa) \rho^{2}
$$

It is obvious that for $\kappa=0$, eqs (21) and (9.b) are fully equivalent.

In the limit cases of $\zeta_{D}=0$ or $\zeta_{D} \rightarrow \infty$ eq (21.c) becomes:

$$
\begin{aligned}
& T_{X K}(0)=\left|\frac{A}{C}\right| \\
& T_{X K}(\infty)=\left|\frac{B}{D}\right|
\end{aligned}
$$

The first step for the optimization procedure, is to identify a pair of frequencies $q_{L}<1$ and $q_{R}>1$ such that:

$T_{X K}\left(q_{L}\right)=T_{X K}\left(q_{R}\right)$

and that the value of $T_{X K}\left(q_{L}\right)=T_{X K}\left(q_{R}\right)$ becomes independent of $\zeta_{D}$. In order that a solution for such a pair of frequencies solution, two alternative conditions must be fulfilled:

Case I:

$A D=B C$

The algebraic elaboration of eq (25) results to:

$\mu\left(q^{2}+\kappa \rho^{2}\right)^{2}=0$

As it can be easily verified, no solution of eq. (26) exists for a positive $q^{2}$, when the values $\mu, \kappa$ and $\rho$ are positive.

Case II:

$A D=-B C$

Elaboration of eq (27) results to:

$(2+\mu) q^{4}-2\left[1+\left(1+\mu+\kappa \mu+\kappa^{2} \mu\right) \rho^{2}\right] q^{2}+\left(2+\kappa^{2} \mu \rho^{2}\right) \rho^{2}=0$

As a result of eq (28) the pair of roots of eq (28) must satisfy:

$q_{L}^{2}+q_{R}^{2}=\frac{2\left[1+\left(1+\mu+\kappa \mu+\kappa^{2} \mu\right) \rho^{2}\right]}{2+\mu}$

Additionally, both roots $q_{L}$ and $q_{R}$ must fulfill eq (23.b), which results to:

$$
\frac{1}{\left.\left(1+\kappa^{2} \mu \rho^{2}\right)-q_{L}^{2}(1+\mu)\right]}=-\frac{1}{\left.\left(1+\kappa^{2} \mu \rho^{2}\right)-q_{R}^{2}(1+\mu)\right]}
$$

or equivalently:

$q_{L}^{2}+q_{R}^{2}=\frac{2\left(1+\kappa^{2} \mu \rho^{2}\right)}{1+\mu}$

The combination of eqs (28) and (30.b) leads to the optimal value of the parameter $\rho$ in terms of the parameters $\kappa$ and $\mu$ : 


$$
\rho_{O P T}=\sqrt{\frac{1}{(1+\mu+\kappa \mu)(1+\mu)-\kappa^{2} \mu}}
$$

As it can be easily verified, eq (31) is reduced to eq (12.a) in case of $\kappa=0$.

Substitution of (31) into eq (28) leads to the pair of values $q_{L}$ and $q_{R}$ :

$$
\begin{aligned}
& q_{L}^{2}=\frac{(1+\mu+\kappa \mu)}{2+\mu}-\frac{\sqrt{\Delta}}{2(2+\mu)} \\
& q_{R}^{2}=\frac{(1+\mu+\kappa \mu)}{2+\mu}+\frac{\sqrt{\Delta}}{2(2+\mu)} \\
& \Delta=4 \frac{2(1+\mu)^{2}(1+\kappa)^{2} \mu(2+\mu)}{\left[(1+\mu+\kappa \mu)(1+\mu)-\kappa^{2} \mu\right]^{2}}
\end{aligned}
$$

Substitution of either (32.a) -or equivalently of(32.b)- into eq (23.b) into (30.a) leads to:

$$
\begin{gathered}
T_{X K L}=T_{X K}\left(q_{L}\right)=T_{X K R}=T_{X K}\left(q_{R}\right)=\sqrt{\frac{2+\mu}{\mu}} \tau(\kappa, \mu)=T_{X M \max } \cdot \tau(\kappa, \mu) \\
\tau(\kappa, \mu)=\frac{(1+\mu+\kappa \mu)(1+\mu)-\kappa^{2} \mu}{(1+\mu)^{2}(1+\kappa)}=\frac{1}{\rho_{O P T}^{2}} \frac{1}{(1+\mu)^{2}(1+\kappa)}
\end{gathered}
$$

As it can be again easily verified, eq (33) is reduced to eq (12) in case of $\kappa=0$.

Considering the selection of $\zeta_{D}$, numerous approaches are possible, the detailed treatment of which is beyond the scope of the current paper. A straightforward approach is followed in the current paper, requiring that for a specified frequency $q_{Z}$ :

$$
T_{X K}\left(q_{Z}\right)=\sqrt{\frac{A^{2}\left(q_{Z}\right)+\left(2 \zeta_{D}\right)^{2} B^{2}\left(q_{Z}\right)}{C^{2}\left(q_{Z}\right)+\left(2 \zeta_{D}\right)^{2} D^{2}\left(q_{Z}\right)}}=T_{X K L} \Leftrightarrow \zeta_{D}=\frac{1}{2} \sqrt{-\frac{C^{2}\left(q_{Z}\right) T_{X K L}^{2}-A^{2}\left(q_{Z}\right)}{D^{2}\left(q_{Z}\right) T_{X K L}^{2}-B^{2}\left(q_{Z}\right)}}
$$

A frequent choice for $q_{S}$ is:

$$
q_{Z}=\sqrt{\rho}
$$

The values of the elements of the KDamper thus finally result as:

$$
\begin{aligned}
& k_{N} / k=\kappa_{\mathrm{N}}=-\kappa \mu \rho^{2} \\
& k_{P} / k=\kappa_{\mathrm{P}}=(1+\kappa) \mu \rho^{2} \\
& k_{S} / k=\kappa_{S}=1+\kappa(1+\kappa) \mu \rho^{2} \\
& m_{D}=\mu m \\
& c_{D}=2 \zeta_{D} \sqrt{\left(k_{P}+k_{N}\right) m_{D}}
\end{aligned}
$$




\section{Basic properties of the KDamper}

Subtraction of the nominator from the denominator in eq (33.b) leads to the following relation:

$(1+\mu)^{2}(1+\kappa)-(1+\mu+\kappa \mu)(1+\mu)+\kappa^{2} \mu=\kappa(1+\mu+\kappa \mu) \geq 0$

Relation (37) implies that:

$1 \geq \tau(\kappa, \mu) \geq 0$

Thus, the following PROPERTY 1 of the KDamper is a direct consequence of eqs (33.a), (38):

PROPERTY 1: The amplitude of the transfer function of the KDamper at the points $q_{L}$ and $q_{R}$ is less than the maximum amplitude of the transfer function of a TMD with equal $\mu$ :

$T_{X K L}=T_{X K R} \leq T_{X M \max }$

The obvious consequence of property 1 is that the addition of a negative stiffness spring reduces the magnitude of the transfer function of the TMD. Figure 4 presents the Transfer function of the KDamper for two values of $\kappa$. An obvious reduction is observed, compared to Fig 3.a.

It should be noted that PROPERTY 1 does not hold for a spring $k_{N}$ with positive stiffness (i.e. with a negative value of $\kappa$ ).

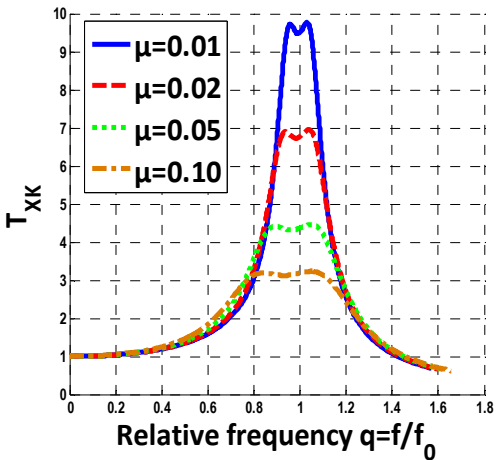

(a)

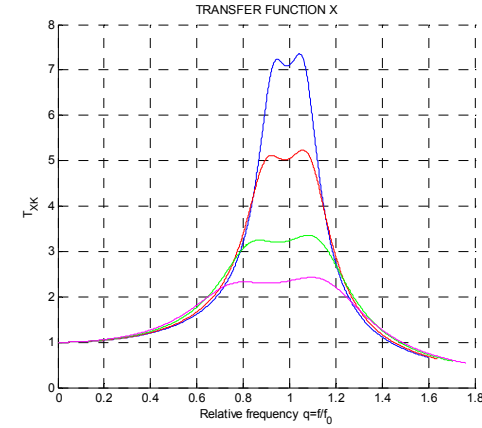

(b)

Figure 4: Transfer function of the KDamper for $(\mathrm{a}) \kappa=0.5$, (b) $\kappa=1.0$

Next, equating the denominator of eq (31) to zero, the following second order equation for $\kappa$ results:

$$
(1+\mu+\kappa \mu)(1+\mu)-\kappa^{2} \mu=0 \Leftrightarrow \kappa^{2} \mu-\kappa \mu(1+\mu)-(1+\mu)^{2}=0
$$

Solution of eq (40) leads to the following maximum value that can be reached for $\kappa$ :

$$
\kappa_{M A X}=(1+\mu) \frac{1+\sqrt{1+4 / \mu}}{2}
$$

The first consequence of eqs (40) and (41) is the following PROPERTY 2. 
PROPERTY 2: The amplitude of the transfer function of the KDamper at the points $q_{L}$ and $q_{R}$ tends to zero when $\kappa$ reaches the limit value of $\kappa_{M A X}$

$T_{X K L}=T_{X K R} \rightarrow 0$ for $\kappa \rightarrow \kappa_{\mathrm{MAX}}$

The most important consequence of eq(42) is that quite small values of the transfer function $T_{X K}$.

Figure 5 presents the variation of $\rho$ and $T_{X K L}=T_{X K R}$ with the increase of the parameter $\kappa$. Figure 6 presents Transfer functions for values of $\kappa$ or $\mu$ close to the limits. Values of low $\mu /$ high $\kappa$ characterize the Kdamper, while high $\mu /$ low $\kappa$ characterize an inerter. As it can be observed, very low values of the transfer function of the KDamper, can be reached, quite below unity. Moreover, these values can be achieved by an almost marginal value of $\mu=0.01$. This fact implies that the KDamper essentially does not require an additional mass $m_{D}$. Moreover, the Kdamper indicates a superior behavior at the very low frequency range.

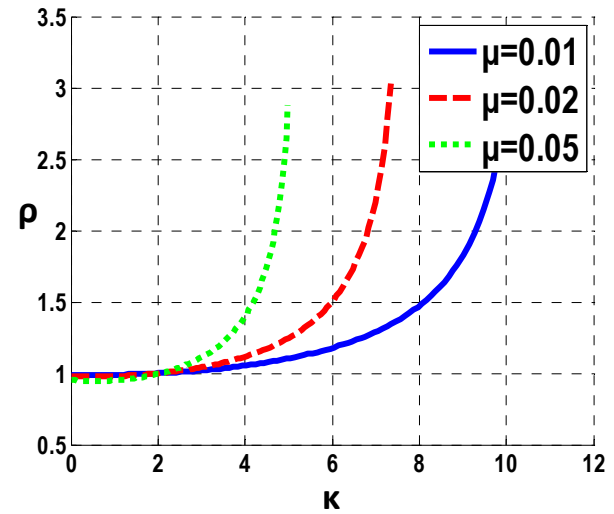

(a)

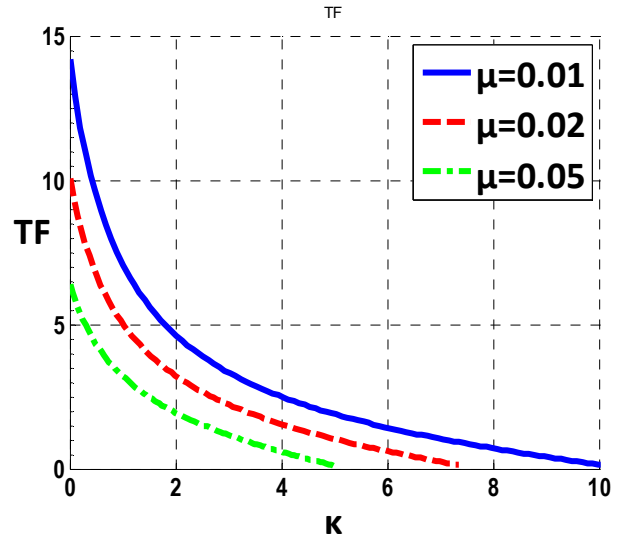

(b)

Figure 5: Variation of the KDamper parameters a) Effect of the mass ratio $\mu$ on the maximum value of $\kappa$. B) Effect of $\kappa$ and $\mu$ over the tolerance parameter $\varepsilon$.

A first implication of of eq (42) is that there exists a range of values of $\kappa$ for which:

$$
T_{X K L}(\kappa)=T_{X K L}(\kappa)<1 \text { for } \kappa_{\mathrm{MAX}} \geq \kappa \geq \kappa_{U N}
$$

Eq. (43) implies that in this case, the values $T_{X K L}=T_{X K R}$ do no longer present the maximum values of $T_{X K}$ since $T_{X K}(q=0)=1$. Among others, this fact complicates the procedures for the selection of $\zeta_{D}$ based on averaging the slopes of $T_{X K}$ at the frequencies $q_{L}, q_{R}$.

Furthermore, although equations (36) and (42) imply that by increasing $\kappa$ the transfer function $T_{X K L}=T_{X K R}$ can be reduced almost to zero, increasing $\kappa$ has a number of implications in the design of 
the KDamper. First, as it can be observed by equations (36.a) to (36.c), high stiffness values result. Figure 7 reflects this fact to the increase of the stiffness values $k_{N}, k_{P}$ and especially $k_{S}$.

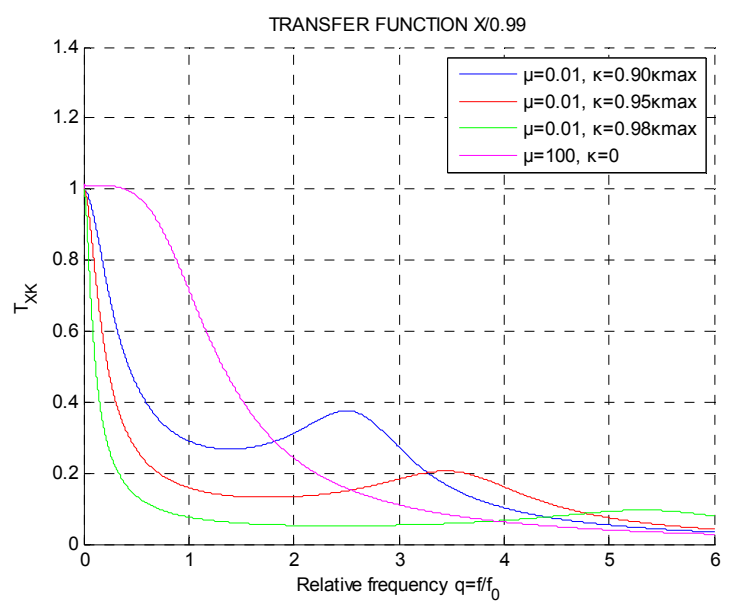

Figure 6: Transfer functions for values of $\kappa$ or $\mu$ close to the limits. Values of low $\mu /$ high $\kappa$ characterize the Kdamper, while high $\mu /$ low $\kappa$ characterize an inerter.

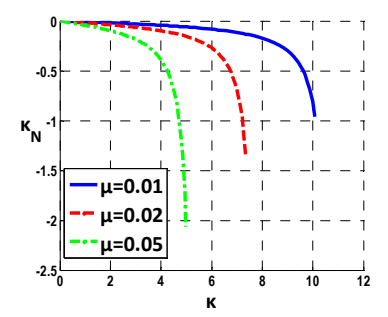

(a)

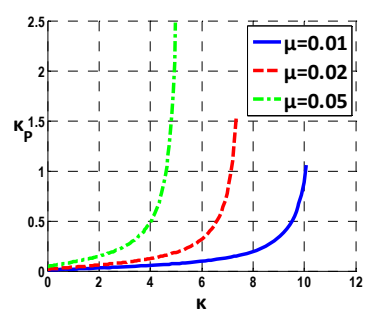

(b)

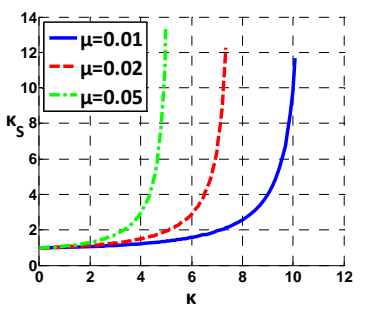

(c)

Figure 7: Increase of the system stiffness by the increase of $\kappa$. (a) $k_{N}$, (b) $k_{P}$, (c) $k_{S}$.

Moreover, increasing the stiffness and especially $k_{N}$ may endanger the static stability of the structure. Although theoretically the value of $k_{N}$ is selected according to eq (14) to ensure the static stability, variations of $k_{N}$ result in practice due to various reasons, such as non-linear behavior, since almost all negative stiffness designs result from unstable non-linear systems, temperature variations, manufacturing tolerances, etc. Consequently, an increase of the absolute value of $k_{N}$ by a factor $\varepsilon$ may lead to a new value of $k_{N L}$ where the structure becomes unstable:

$k_{S}+\frac{k_{P} k_{N L}}{k_{P}+k_{N L}}=0 \Leftrightarrow k_{N L}=-\frac{k_{S} k_{P}}{k_{S}+k_{P}}=(1+\varepsilon) k_{N}$

Substitution of (36.a) to (36.c) into (44) leads to the following estimate for the static stability margin $\varepsilon$ : 
$\varepsilon=\frac{1}{\kappa\left[\left(1+(1+\kappa)^{2} \mu \rho^{2}\right]\right.}$

As it can be shown, the following PROPERTY 3 holds for $\varepsilon$.

PROPERTY 3: The increase of the negative stiffness of the system is upper bounded by the static stability limit of the structure:

$\varepsilon \rightarrow 0$ for $\kappa \rightarrow \kappa_{\mathrm{MAX}}$

Figure 8 presents the variation of $\kappa_{M A X}$ over $\mu$ and of $\varepsilon$ over $\kappa$ and $\mu$.

Finally, as it is observed from eq. (21.d), high values of $\kappa$ result to increased amplitudes of the response $y$, which may encounter further technological constraints. For example, eq (21.d) for $q=0$ leads to:

$T_{Y X K}(q=0)=\frac{Y(q=0)}{X(q=0)}=\frac{Y(q=0)}{X_{S T}}=1+\kappa$

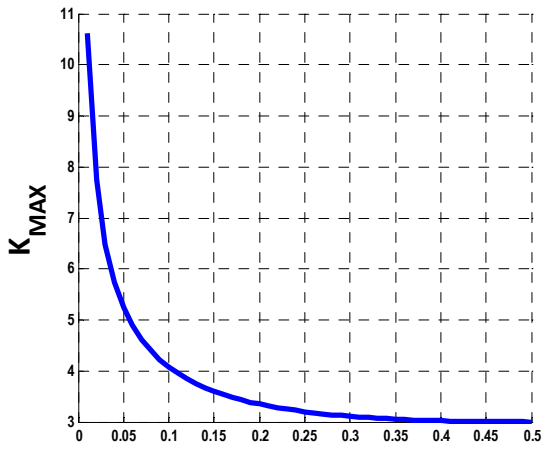

(a)

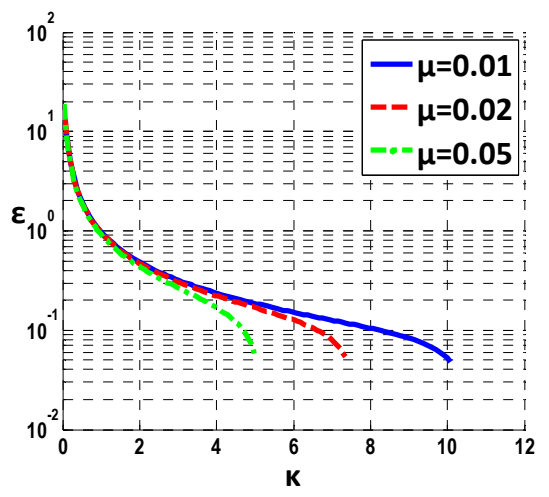

(b)

Figure 8: Variation of KDamper parameters a) Effect of the mass ratio $\mu$ on the maximum value of $\kappa$. B) Effect of $\kappa$ and $\mu$ over the static stability margin $\varepsilon$.

\section{An implementation example}

\subsection{Indicative realization of a negative stiffness spring}

An example for an implementation of the KDamper is depicted in Figure 9. It consists from a mass $m$ which is supported by two parallel linear springs with stiffness $k_{S}$ and $k_{P}$ respectively and by a damper with constant $c_{D}$. The damper $c_{D}$ and the spring $k_{P}$ are in turn are connected to a mass $m_{D}$. The negative stiffness spring is realized by a set of two symmetric linear horizontal springs with constants $\mathrm{k}_{\mathrm{H}}$, which support the mass $m_{D}$ by an articulated mechanism. 
The static equilibrium position of the system is depicted in Fig 9(a), under the action of the gravity force. The perturbed position after an external dynamic excitation $f(t)$ is depicted in Fig 9(b), along with the necessary notation concerning the various displacements of the system.

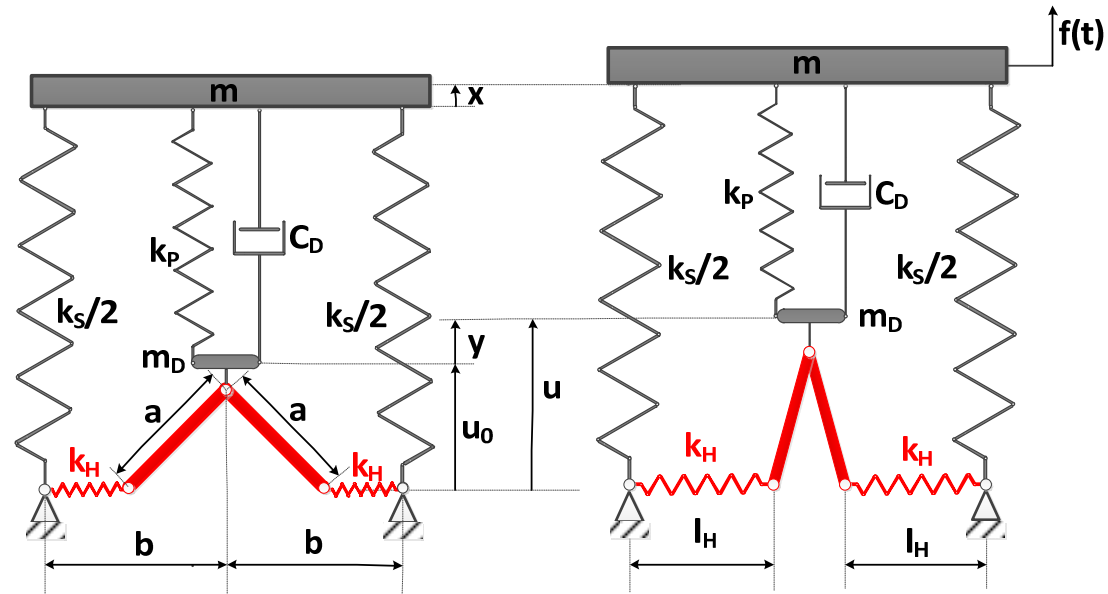

(a)

(b)

Figure 9: Variation of the KDamper parameters a) Effect of the mass ratio $\mu$ on the maximum value of $\kappa$. B) Effect of $\kappa$ and $\mu$ over the tolerance parameter $\varepsilon$.

The equations of motion of the proposed oscillator are:

$$
\begin{aligned}
& m \ddot{x}+c_{D}(\dot{x}-\dot{y})+k_{S}\left(l_{S}-l_{S I}\right)+k_{P}\left(l_{P}-l_{P I}\right)=f+m g \\
& m_{D} \ddot{y}-c_{D}(\dot{x}-\dot{y})-k_{P}\left(l_{P}-l_{P I}\right)+f_{N}(u)=m_{D} g
\end{aligned}
$$

where $l_{S}(\mathrm{t})$ is the length of the spring $k_{S}, l_{S I}$ is the initial length of the un-deformed spring $k_{S}, l_{P}(\mathrm{t})$ is the length of the spring $k_{P}, l_{P I}$ is the initial length of the un-deformed spring $k_{P}$ and $f_{N}(u)$ is the nonlinear force exerted by the set of the two symmetric oblique springs $k_{H}$.

The equations of the system at the static equilibrium point are derived by the equations (47):

$$
\begin{aligned}
& k_{S}\left(l_{S 0}-l_{S I}\right)+k_{P}\left(l_{P 0}-l_{P I}\right)=m g \\
& -k_{P}\left(l_{P 0}-l_{P I}\right)+f_{N}\left(u_{0}\right)=m_{D} g
\end{aligned}
$$

where the index ()$_{0}$ is used to denote the static equilibrium point, $l_{S 0}$ is the length of the (normally deformed) spring $k_{S}$ at the static equilibrium point, $l_{P 0}$ is the length of the (normally deformed) spring $k_{P}$ at the static equilibrium point and $f_{N}\left(u_{0}\right)$ is the non-linear force exerted by the set of the two symmetric oblique springs $k_{H}$ at the static equilibrium point.

Denoting by: 


$$
\begin{aligned}
& v_{S}=l_{S 0}-l_{S I} \\
& v_{P}=l_{P 0}-l_{P I} \\
& f_{N 0}=f_{N}\left(u_{0}\right)
\end{aligned}
$$

the following equations result:

$$
\begin{aligned}
& v_{S}=\left[\left(m+m_{D}\right) g-f_{N 0}\right] / k_{S} \\
& v_{E}=\left(-m_{D} g+f_{N 0}\right) / k_{P}
\end{aligned}
$$

Further elaboration of the sets of Eqs (48),(49),(50) and substitution in the set of eqs (47) leads to the final set of equations of motion:

$$
\begin{aligned}
& m \ddot{x}+c_{D}(\dot{x}-\dot{y})+k_{S} x+k_{P}(x-y)=f \\
& m_{D} \ddot{y}-c_{D}(\dot{x}-\dot{y})-k_{P}(x-y)+f_{N D}(u)=0
\end{aligned}
$$

where:

$$
\begin{aligned}
& f_{N D}(u)=f_{N}(u)-f_{N 0} \\
& u=u_{0}+y \\
& l_{S}=l_{S 0}+x \\
& l_{P}=l_{P 0}+x-y
\end{aligned}
$$

The following expressions can be derived for the potential energy $U_{N}$, the non-linear force $f_{N}$ and the equivalent non-liner stiffness $k_{N}$ of the set of the horizontal springs $k_{H}$ :

$$
\begin{aligned}
& U_{N}[u(y)]=2 \frac{1}{2} k_{H}\left(l_{H}-l_{H I}\right)^{2} \\
& f_{N}(u)=\frac{\partial U_{N}}{\partial y}=\frac{\partial U_{N}}{\partial u}=-2 k_{H}\left(1+\frac{l_{H I}-b}{\sqrt{a^{2}-u^{2}}}\right) u=-2 k_{H}\left(1+c_{I} \frac{1}{\left(1-u^{2} / a^{2}\right)^{1 / 2}}\right) u \\
& k_{N}=\frac{\partial f_{N}}{\partial y}=\frac{\partial f_{N}}{\partial u}=-2 k_{H}\left[1+\frac{l_{H I}-b}{a} \frac{1}{\left(1-u^{2} / a^{2}\right)^{3 / 2}}\right]=-2 k_{H}\left[1+c_{I} \frac{1}{\left(1-u^{2} / a^{2}\right)^{3 / 2}}\right]
\end{aligned}
$$

where $l_{H I}$ is the initial length of the un-deformed springs $k_{H}, l_{H}(t)$ is the length of the springs $k_{H}$ :

$$
l_{H}=b-\left(\alpha^{2}-u^{2}\right)^{1 / 2}
$$

and:

$$
c_{I}=\frac{l_{H I}-b}{a}
$$

In the case of $c_{\Gamma}=0$ the two horizontal springs are equivalent to a spring with a constant negative stiffness of $k_{N}=-2 k_{H}$ 


\subsection{Selection of the system parameters}

The vibrations of a mass of $\mathrm{m}=500 \mathrm{Kg}$ are to be isolated, which is supported in the vertical direction by a system of springs of with a total static stiffness of $k=2.4 \times 10^{6} \mathrm{~N} / \mathrm{m}$. The corresponding vertical static deflection of the system under its own weight is $X_{V S D}=2 \mathrm{~cm}$ and the natural frequency of the system is $f_{0}=3.5 \mathrm{~Hz}$. The maximum permissible equivalent static amplitude of the vibrations of the mass is $X_{S T}=1 \mathrm{~cm}$.

The main parameters of a KDamper system are selected as $\mu=0.01$ and $\kappa=7.8$. The resulting static stability margin is $\varepsilon=0.05$. The full set of the KDamper parameters is presented in Tables 1 and 2 and the resulting transfer functions for the displacements $x$ and $y$ are presented in Fig 10.

Table 1. Non-dimensional KDamper parameters

\begin{tabular}{|c|c|c|c|c|c|c|c|c|c|}
\hline$\mu$ & $\kappa$ & $\mathrm{E}$ & $\rho$ & $\zeta_{D}$ & $q_{L}$ & $q_{R}$ & $\kappa_{N}$ & $\kappa_{P}$ & $\kappa_{S}$ \\
\hline 0.01 & 7.8 & 0.05 & 1.4279 & 0.69 & 0.97 & 1.87 & -0.159 & 0.1784 & 2.400 \\
\hline
\end{tabular}

Table 2. Dimensional KDamper parameters

\begin{tabular}{|c|c|c|c|c|}
\hline$k_{S}$ & $k_{P}$ & $k_{N}$ & $m_{D}$ & $c_{D}$ \\
\hline $5.8587 \times 10^{5} \mathrm{~N} / \mathrm{m}$ & $4.306 \times 10^{4} \mathrm{~N} / \mathrm{m}$ & $-3.8167 \times 10^{4} \mathrm{~N} / \mathrm{m}$ & $5.0 \mathrm{Kg}$ & $215.87 \mathrm{Nsec} / \mathrm{m}$ \\
\hline
\end{tabular}

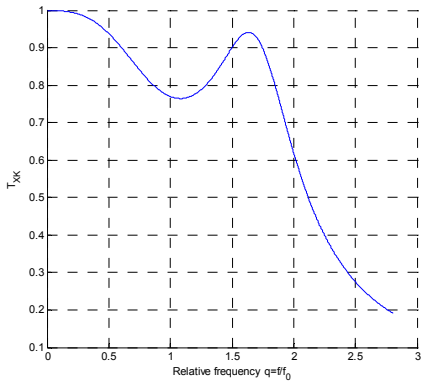

(a)

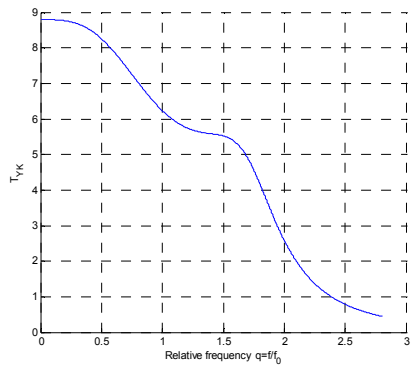

(b)

Figure 10: Transfer functions of the KDamper used in the application example (a) $T_{X K}$, (b) $T_{Y K}$.

Next, the parameters of the negative stiffness spring and the corresponding mechanism are selected. In view of Fig $10 \mathrm{~b}$ and taking into account that the maximum permissible equivalent static amplitude of the vibrations of the mass is $X_{S T}=1 \mathrm{~cm}$, a maximum amplitude of $u_{M}=10 \mathrm{~cm}$ is selected. The value of $u_{0}$ is selected as $u_{0}=0.1 \mathrm{~cm}$. This value is selected close to $u=0$, so that an almost symmetric response around $u=0$ is obtained.

The rest of the parameters of the oblique springs are selected so that $k_{N}(u=0)=1.01 k_{N C}$ and $k_{N}\left(u_{M}\right)=0.90 k_{N C}$, where $k_{N C}=-3.8167 \times 10^{4} \mathrm{~N} / \mathrm{m}$ is the constant negative stiffness of the KDamper in Table 2. Since $k_{N}(u=0)$ is the minimum value of $k_{N}$, this setting of $k_{N}(u=0)$ guarantees that in view of 
eq (14), the system of springs remains statically stable for the entire operating range. Parallel, the choice of $k_{N}\left(u_{M}\right)$ guarantees that $k_{N}(u)$ retains a sufficient level of negative values in the entire operating range, so that the damping properties of the oscillator are not compromised. A small negative value for $c_{I}=-0.05$ is selected, in order to guarantee as far as possible a linear behaviour will be assumed. The resulting parameters of the negative stiffness springs and mechanism are $k_{H}$ $=2.0289 \times 10^{4} \mathrm{~N} / \mathrm{m}, l_{H I}=0.430 \mathrm{~m}, \mathrm{a}=0.124 \mathrm{~m}, \mathrm{~b}=0.4367 \mathrm{~m}$.

Table 3. Negative stiffness spring and mechanism parameters

\begin{tabular}{|c|c|c|c|c|}
\hline$k_{H}$ & $l_{H I}$ & $a$ & $b$ & $u_{0}$ \\
\hline $2.0289 \times 10^{4} \mathrm{~N} / \mathrm{m}$ & $0.430 \mathrm{~m}$ & $0.124 \mathrm{~m}$ & $0.4367 \mathrm{~m}$ & $0.1 \mathrm{~cm}$ \\
\hline
\end{tabular}

\subsection{Response analysis}

The Transfer Functions (Frequency Response Functions) $T_{X K}, T_{Y K}$ of the displacements $x$ and y of the proposed non-linear oscillator to a harmonic excitation force with an amplitude of $F_{e}=F_{M A X}=k X_{S T}=2400 \mathrm{~N}$ are presented in Fig 11 .

The transfer functions are in a very good agreement to that of the reference linear stiffness oscillator.

Figure 12 presents the waveforms of the displacements and velocities of the response of the nonlinear oscillator to the harmonic excitation force of $F_{e}=F_{M A X}=2400 \mathrm{~N}$ for two different excitation frequencies of $f_{e}=f_{0}=3.48 \mathrm{~Hz}$ and of $f_{e}=1.0 \mathrm{~Hz}$, where the maximum response amplitude is observed. The response at $f_{e}=f_{0}=3.48 \mathrm{~Hz}$ is essentially a harmonic waveform.

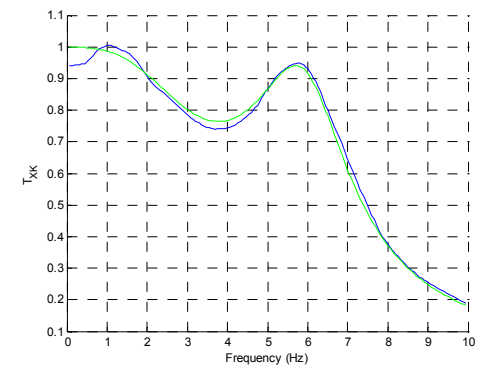

(a)

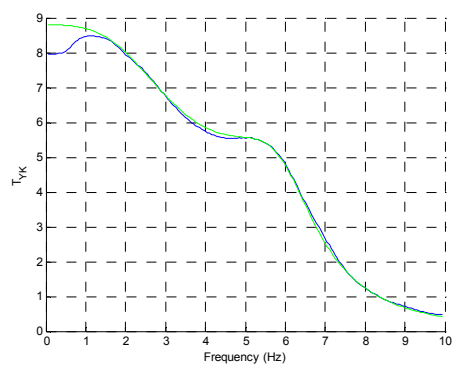

(b)

Figure 11 Frequency Response Functions of the proposed oscillator to a harmonic excitation force of $F_{e}=F_{M A X}=0.05 \mathrm{~N}$ a)Displacement $\mathrm{x}, \mathrm{b}$ ) Forces ['b'=nonlinear]

Contrary, the response at the low frequency excitation of $f_{e}=1.0 \mathrm{~Hz}$ indicates characteristic nonlinear features. The presence of non-linear features is due to the non-linear nature of the negative 
stiffness force, which is dominant over inertia forces at the low frequency range, which indicates that further analysis is necessary for the nature and characteristics of this non-linear response in the low frequency range. Among others, these non-linear features can be further exploited in order to enhance the vibration absorption features of the KDamper, especially in the highly demanding lowfrequency range
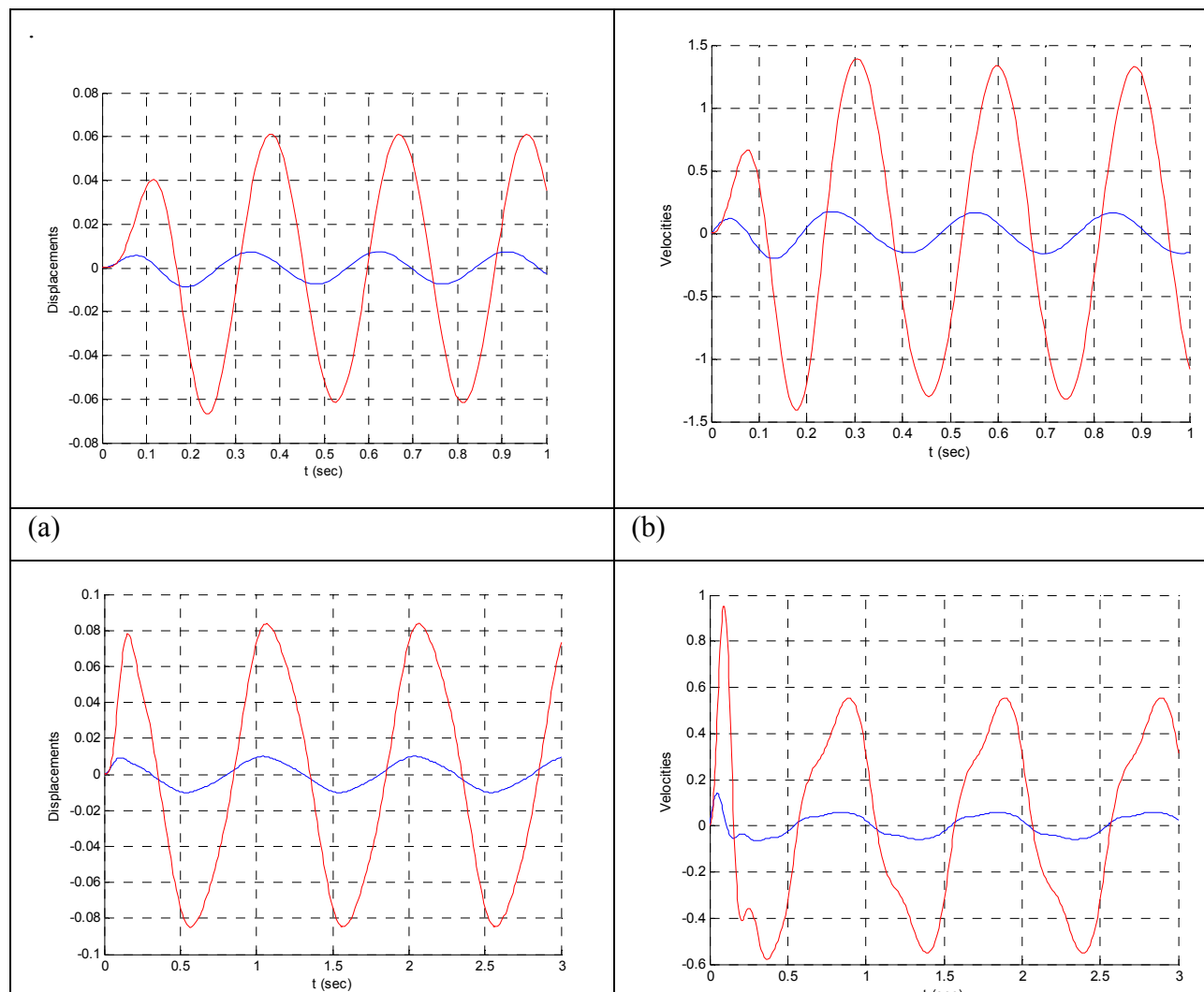

(b)

(c)

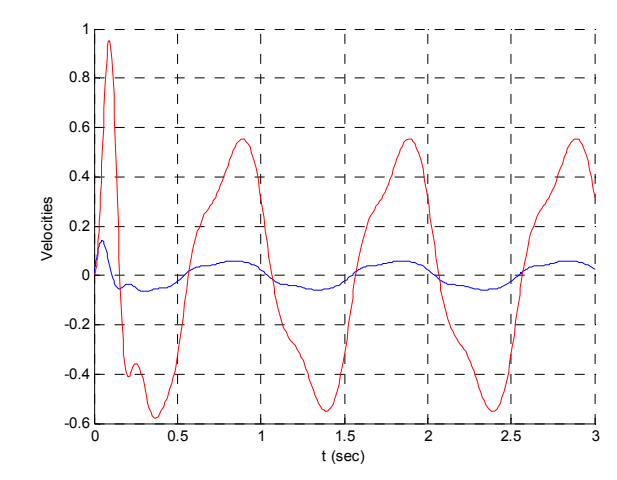

(d)

Figure 12 Response of the non-linear oscillator to a harmonic excitation force of $F_{e}=F_{M A X}=k X_{S T}=2400 \mathrm{~N}$ (a) Displacements, $f_{e}=3.48 \mathrm{~Hz}$ (b) Velocities, $f_{e}=3.48 \mathrm{~Hz}$, (c) Displacements, $f_{e}=1.0 \mathrm{~Hz}(\mathrm{~d})$ Velocities, $f_{e}=1.0 \mathrm{~Hz} \cdot(\mathrm{b}=\mathrm{x}, \mathrm{r}=\mathrm{y})$

Figure 13a presents the variation of $k_{N}$ over time and Fig 13.b as a function of the displacement $u$ of the set of the negative stiffness springs. A significant variation of $k_{N}$ is observed, verifying the strong no-linear nature of the response. However, the negative stiffness $k_{N}$ remains within the specified acceptable limits, which guarantee both static stability and damping behaviour. 


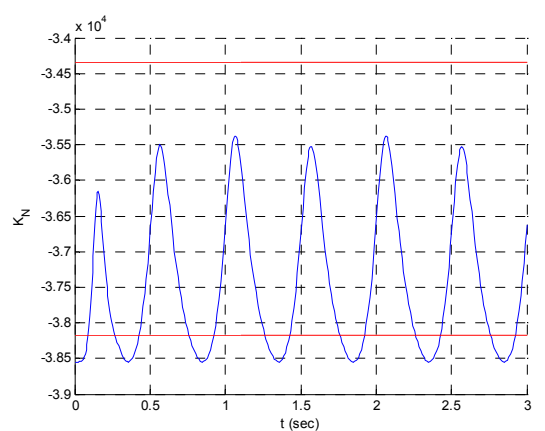

(a)

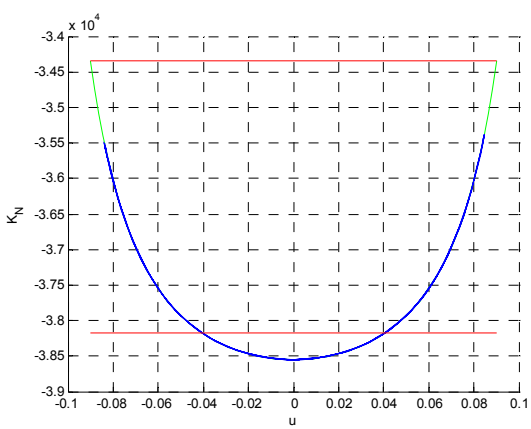

(b)

Figure 13. Variation of the non-linear stiffness $k_{N}$ to a harmonic excitation force of $F_{e}=2400 \mathrm{~N}$ and $f_{e}=1.0 \mathrm{~Hz}$ :. a) Variation in the time domain. b) Range of oscillation.

\subsection{Balance of Forces}

Further insight to the physical behavior of the system is provided, by analyzing the balance of forces, as expressed by eqs (51). Figure 14 presents the magnitudes, the real and the imaginary parts of the Frequency Response Functions of the forces in the entire frequency range. As it is observed from the balance of forces acting on the mass $m$, (left column in Fig 14), the external force $\mathrm{Fe}$ is almost entirely balanced by the positive stiffness force $F_{S}$ and negative stiffness force $F_{N D}$, as well as by the inertia force $F_{M}$ of the main mass $m$. The balance of forces on the additional mass $m_{D}$ (right column in Fig 14) indicates that the negative stiffness force $F_{N D}$ is essentially balanced by the positive stiffness force $F_{P}$.

The magnitude of the damping force $F_{C D}$ and of the inertia force $F_{M D}$ of the additional mass, is minimal throughout the entire frequency range, including the natural frequencies of the system. Contrary to the conventional linear dynamic systems, where the damping forces essentially peak at the resonance and thus balance the external forces, the effect of the damper in the KDamper is to generate a proper phase difference phase between the positive stiffness force $F_{S}$ and the negative stiffness force $F_{N D}$.

This phase difference is more evident in Fig. 15, which presents the waveforms of the forces at three characteristic excitation frequencies. This fact ensures that an adequate level of elastic forces exists throughout the entire frequency range, able to counteract the inertial and the external excitation forces and provides further insight on the background of stiffness based vibration isolation. 


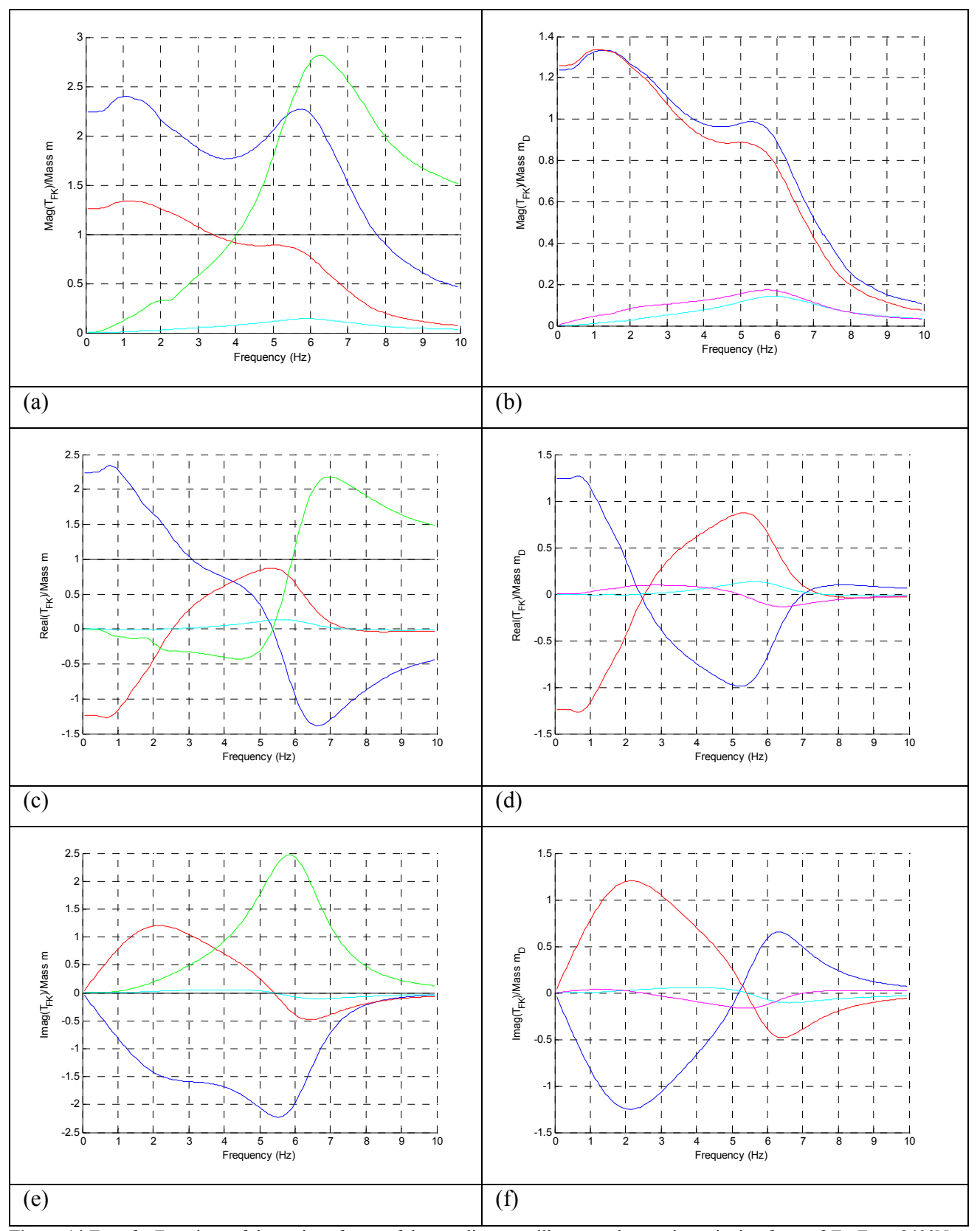

Figure 14 Transfer Functions of the various forces of the non-linear oscillator to a harmonic excitation force of $F_{e}=F_{M A X}=2400 \mathrm{~N}$ : a)Magnitudes of TFs for the forces at the mass $m, \mathrm{~b}$ ) Magnitudes of TFs for the forces at the mass $m_{D}$, c)Real parts of the TFs for the forces at the mass $m$, d)Real parts of the TFs for the forces at the mass $m_{D}$ e)Imaginary parts of the TFs for the forces at the mass $m$, f)Imaginary parts of the TFs for the forces at the mass $m_{D .}$. $[\mathrm{b}=\mathrm{FS}$ or FP, $\mathrm{r}=\mathrm{FN}, \mathrm{g}=\mathrm{FM}, \mathrm{c}=\mathrm{FMD}, \mathrm{m}=\mathrm{FCD}, \mathrm{b}=\mathrm{Fe}]$ 


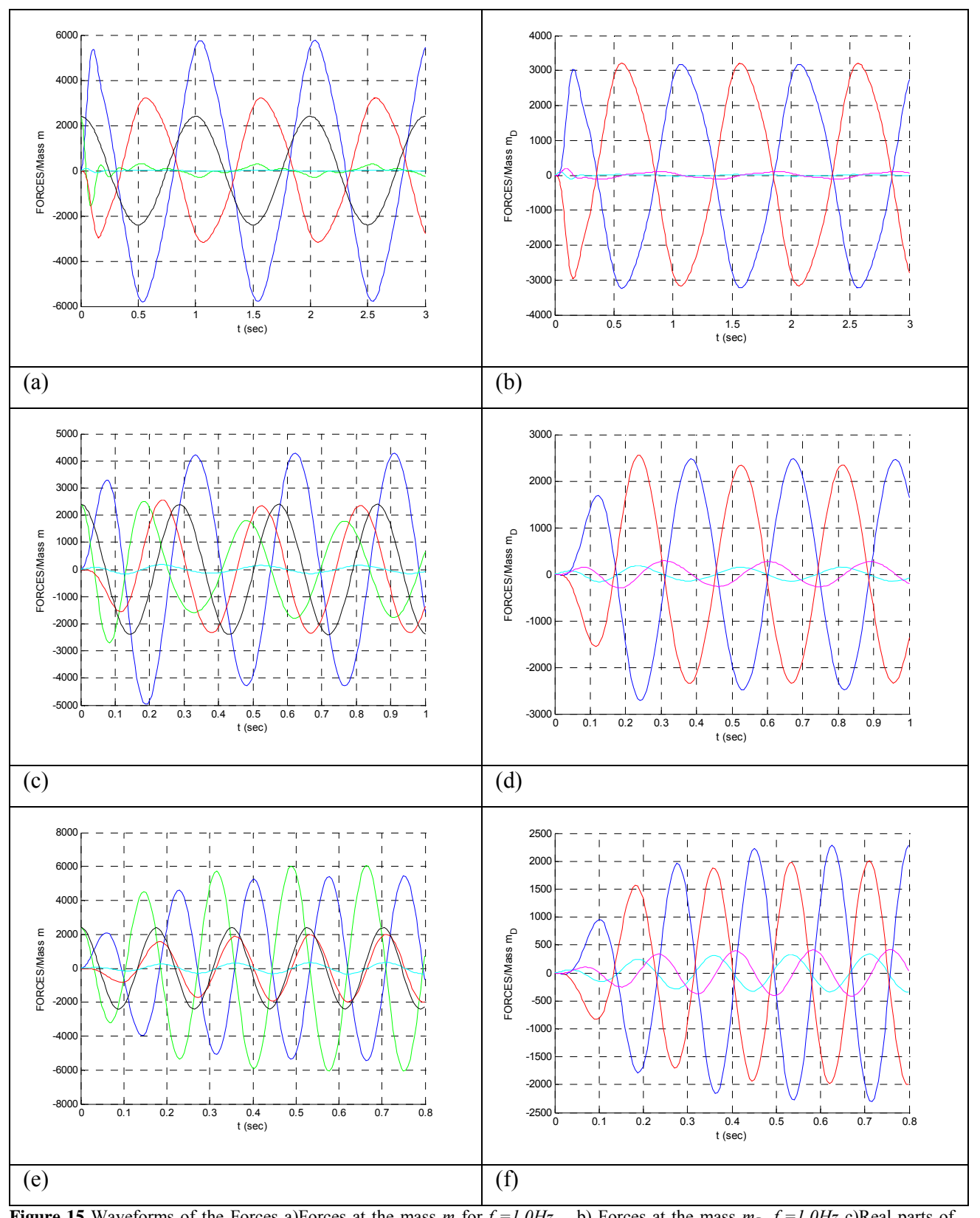

Figure 15 Waveforms of the Forces a)Forces at the mass $m$ for $f_{e}=1.0 \mathrm{~Hz}$, b) Forces at the mass $m_{D}, f_{e}=1.0 \mathrm{~Hz}$ c)Real parts of Transfer Functions for the forces at the mass $m, f_{e}=3.48 \mathrm{~Hz}$ d)Real parts of Transfer Functions for the forces at the mass $m_{D}$ $f_{e}=3.48 \mathrm{~Hz}$ e)Imaginary parts of Transfer Functions for the forces at the mass $m$, for $f_{e}=5.7 \mathrm{~Hz}$ ) Imaginary parts of Transfer Functions for the forces at the mass $m_{D} f_{e}=5.7 \mathrm{~Hz}[\mathrm{~b}=\mathrm{FS}$ or $\mathrm{FP}, \mathrm{r}=\mathrm{FN}, \mathrm{g}=\mathrm{FM}, \mathrm{c}=\mathrm{FMD}, \mathrm{m}=\mathrm{FCD}, \mathrm{b}=\mathrm{Fe}]$ 


\section{Conclusion}

The systematic design procedure of section 3 leads to a KDamper performance that can inherently offer far better isolation and damping properties than the TMD. Moreover, since the isolation and damping properties of the $\mathrm{KD}$ essentially result from the stiffness elements of the system, further technological advantages can emerge over JD, in terms of weight, complexity and reliability, without any need for compromises in the overall stiffness of the structure as it is the case of QZS.

The background of the performance of this stiffness based vibration absorption concept is the fact that an adequate level of elastic forces exists throughout the entire frequency range, able to counteract the inertial and the external excitation forces, while the damping forces and the inertia forces of the additional mass remain minimal in the entire frequency range, including the natural frequencies.

Moreover, the inherent non-linear nature of the negative stiffness force can be exploited to offer further potential advantages of the KDamper concept, such as robustness, broadband response and energy sink.

Such a dam oscillator concept presents the potential for numerous implementations in a large variety of technological applications, either as a discrete vibration isolator, or in the form of periodic metamaterials and composite structures. Moreover, further applications may emerge in a multi- physics environment.

\section{References}

[1] Frahm, H., 1909, Device for Damping Vibrations of Bodies, , US patent \#989958,

[2] Ormondroyd, J. and Den Hartog, J. P. (1928) The Theory of Dynamic Vibration Absorber, Trans. ASME, APM-50-7, P. P. 9-22.

[3] Den Hartog, JP.,Mechanical Vibrations (4th edition), McGraw-Hill, New York;(1956).

[4]Nirmalendu Debnath, SK Deb and Anjan Dutta, 2015, Multi-modal vibration control of truss bridges with tuned mass dampers under general loading, Journal of Vibration and Control, DOI: $10.1177 / 1077546315571172$

[5]Jun-Ling Chen1 and Christos T Georgakis, 2015, Spherical tuned liquid damper for vibration control in wind turbines, Journal of Vibration and Control, 2015, Vol. 21(10) 1875-1885

[6] J. Mayet, H.Ulbrich, 2015, First-order optimal linear and nonlinear detuning of centrifugal pendulum vibration absorbers, Journal ofSoundandVibration335(2015)34-54

[7], Amir Younespour and Hosein Ghaffarzadeh, Structural active vibration control using active mass damper by block pulse functions, Journal of Vibration and Control 2015, Vol. 21(14) 2787-2795. 
[8]M.C. Smith, Synthesis of mechanical networks: The Inerter, IEEE, Trans. on Automatic Control, vol. 47, 2002, pp. 1648-1662.

[9] M.Z.Q. Chen and M.C. Smith. Restricted complexity network realizations for passive mechanical control. IEEE Trans. on Automatic Control, 54(10):2290-2301, 2009.

[10] M.Z.Q. Chen, C. Papageorgiou, F. Scheibe, Fu cheng Wang, and M.C. Smith. The missing mechanical circuit element. Circuits and Systems Magazine, IEEE, 9(1):10_26, 2009.

[11] F.-C. Wang, M.-R. Hsieh, H.-J. Chen, Stability and performance analysis of a full-train system with inerters. Vehicle System Dynamics, 50(4):545_571, 2012.

[12] I. Takewaki, S. Murakami, S. Yoshitomi, and M. Tsuji. Fundamental mechanism of earthquake response reduction in building structures with inertial dampers. Structural Control and Health Monitoring, 19(6):590_608, 2012.

[13],Chuan Li, Ming Liang, Yixian Wang and Yutao Dong, 2012, Vibration suppression using twoterminal flywheel. Part I: Modeling and characterization, Journal of Vibration and Control 18(8) 1096-1105.

[14], Fu-Cheng Wang and Sheng-Yao Wu, 2015, Vibration control of an optical table employing mechatronic inerter networks, Journal of Vibration and Control, DOI: 10.1177/1077546314528365

[15] P. Brzeski, T. Kapitaniak, P. Perlikowski, 2015, Novel type of tuned mass damper with inerter which enables changes of inertance, Novel type of tuned mass damper with inerter which enables changes of inertance, Journal of Sound and Vibration, Volume 349, 4 2015, Pages 56-66.

[16] W. Molyneaux, Supports for vibration isolation, ARC/CP-322, Aero- nautical Research Council, Great Britain, 1957.

[17] D. L. Platus, Negative-stiffness-mechanism vibration isolation systems, In: SPIE's International Symposium on Optical Science, Engineering, and Instrumentation (1999) 98 105.

[18] A. Carrella, M. Brennan, T. Waters, Static analysis of a passive vibration isolator with quasi-zero-stiffness characteristic, Journal of Sound and Vibration 301 (2007) 678-89.

[19] R. Ibrahim, 2008, Recent advances in nonlinear passive vibration isolators, Journal of Sound and Vibration 314 (2008) 371-452.

[20] J. Winterflood, D.Blair, B.Slagmolen, High performance vibration isolation using springs in Euler column buckling mode, Physics Letters A 300(2002) 122-130.

[21] L. Virgin, S.Santillan, R.Plaut, Vibration isolation using extreme geometric nonlinearity, Journal of Sound and Vibration 315 (2008)721-731.

[22] A.V.Dyskin, E.Pasternak, 2012, Mechanical effect of rotating non-spherical particles on failure in compression, Philosophical Magazine 92 (2012)3451-3473.

[23] Will S.Robertson, M.R.F.Kidner, BenS.Cazzolato, AnthonyC.Zander, 2009, Theoretical design parameters for a quasi-zero stiffness magnetic spring for vibration isolation, Journal of Sound and Vibration 326 (2009) 88-103.

[24] N. Zhou, K.Liu, 2010, A tunable high-static-low-dynamic stiffness vibration isolator, Journal of Sound and Vibration 329 (2010) 1254-1273.

[25] R. DeSalvo, Passive, nonlinear, mechanical structures for seismic attenuation, Journal of Computational and Nonlinear Dynamics 2 (2007)290-298. 
[26] H. Iemura, M. H. Pradono, Advances in the development of pseudo- negative-stiffness dampers for seismic response control, Structural Control and Health Monitoring 16 (2009) 784 99.

[27] A. A. Sarlis, D. T. R. Pasala, M. Constantinou, A. Reinhorn, S. Nagarajaiah, D. Taylor, Negative stiffness device for seismic protection of structures, Journal of Structural Engineering 139 (2012) 1124-33.

[28] Navid Attary, M Symans, S Nagarajaiah, 2015, Development of a rotation-based negative stiffness device for seismic protection of structures, Journal of Vibration and Control, DOI: $10.1177 / 1077546315585435$.

[29] C.M. Lee, V. Goverdovskiy, A. Temnikov, Design of springs with negative stiffness to improve vehicle driver vibration isolation, Journal of Sound and Vibration 302 (2007) 86574.

[30] T. D. Le, K. K. Ahn, A vibration isolation system in low frequency excitation region using negative stiffness structure for vehicle seat, Journal of Sound and Vibration 330 (2011) 631135 .

[31] C.-M. Lee, V. Goverdovskiy, A multi-stage high-speed railroad vibration isolation system with negative stiffness, Journal of Sound and Vibration, 331 (2012) 914-21.

[32] Jiaxi Zhou, Daolin Xu, Steven Bishop, 2015, A torsion quasi-zero stiffness vibration isolator, Journal of Sound and Vibration 338 121-133.

[33] K. Virk, A. Monti, T. Trehard, M. Marsh, K. Hazra, K. Boba, C. Remillat, F. Scarpa, I. Farrow, SILICOMB PEEK Kirigami cellular structures : mechanical response and energy dissipation through zero and negative stiffness, Smart Materials and Structures 22 (2013) 084014-.

[34] E. Baravelli, M. Ruzzene, Internally resonating lattices for bandgap generation and lowfrequency vibration control, Journal of Sound and Vibration 332 (2013) 6562-79.

[35] P. Michelis, V. Spitas, Numerical and experimental analysis of a tri- angular auxetic core made of cfr-peek using the directionally reinforced integrated single-yarn (DIRIS) architecture, Composites Science and Technology 70 (2010) 1064-71.

[36] Dixon M. Correa, Timothy Klatt, Sergio Cortes, Michael Haberman, Desiderio Kovar and Carolyn Seepersad, 2015, Negative stiffness honeycombs for recoverable shock isolation, Rapid Prototyping Journal 21/2 (2015) 193-200.

[37] R. Lakes, P. Rosakis, A. Ruina, Microbuckling instability in elastomeric cellular solids, Journal of Materials Science 28 (1993) 4667-72.

[38] V. Spitas, C. Spitas, P. Michelis, Modeling of the elastic damping res- ponse of a carbon nanotube-polymer nano-composite in the stress-strain domain using an elastic energy release approach based on stick-slip, Mechanics of Advanced Materials and Structures 20 (2013) 791800 .

[39] D.I.Chortis, D.S.Varelis, D.A.Saravanos, 2013, "Linearized Frequencies and Damping in Composite Laminated Beams Subject to buckling, Journal of Vibration and Acoustics, Transactions of the ASME, April 2013, V135, 021006-1-10.

[40] R. Lakes, Extreme damping in composite materials with a negative stiffness phase, Physical Review Letters 86 (2001) 2897-8. 
[41] T. Jaglinski, D. Kochmann, D. Stone, R. Lakes, Composite materials with viscoelastic stiffness greater than diamond, Science 315 (2007), 620-2.

[42] L. Dong, R. Lakes, Advanced damper with high stiffness and high hysteresis damping based on negative structural stiffness, International Journal of Solids and Structures 50 (2013) 2416-23.

[43] C. S. Wojnar, D. M. Kochmann, A negative-stiffness phase in elastic composites can produce stable extreme effective dynamic but not static stiffness, Philosophical Magazine 94 (2014) 532-55.

[44], Vakakis AF, Gendelman OV, Bergman LA, McFarland DM, Kerschen G and Lee YS (2008) Nonlinear Targeted Energy Transfer in Mechanical and Structural Systems I. Berlin: Springer.

[45] Maor Farid and Oleg V Gendelman, 2015, Tuned pendulum as nonlinear energy sink for broad energy range, Journal of Vibration and Control, DOI: 10.1177/1077546315578561.

[46] C.-M. Lee, V. Goverdovskiy, S. Samoilenko, Prediction of non-chaotic motion of the elastic system with small stiffness, Journal of Sound and Vibration 272 (2004) 643-55.

[47] A. Carrella, M. Brennan, T. Waters, V. Lopes Jr, Force and displacement transmissibility of a nonlinear isolator with high-static-low- dynamic-stiffness, International Journal of Mechanical Sciences 55 (2012) 22-9.

[48] I. Kovacic, M. J. Brennan, T. P. Waters, A study of a nonlinear vibration isolator with a quasi-zero stiffness characteristic, Journal of Sound and Vibration 315 (2008) 700-11.

[49] A. Shaw, S. Neild, D. Wagg, Dynamic analysis of high static low dynamic stiffness vibration isolation mounts, Journal of Sound Vibration, 332 (2013) 1437-55.

[50] I. Antoniadis, D. Chronopoulos, V. Spitas, D. Koulocheris, "Hyper-damping properties of a stable linear oscillator with a negative stiffness element", Journal of Sound and Vibration, 2015, V346, pp 37-52.

[51] D. Chronopoulos, I. Antoniadis, M. Collet, M. Ichchou, "Enhancement of wave damping within metamaterials having embedded negative stiffness inclusions", 2015, Wave Motion, http://dx.doi.org/10.1016/j.wavemoti.2015.05.005. 